\title{
Symmetry and transport property of spin current induced spin-Hall effect
}

\author{
Yanxia Xing, ${ }^{1}$ Qing-feng Sun, ${ }^{1, *}$ and Jian Wang ${ }^{2}$ \\ ${ }^{1}$ Beijing National Laboratory for Condensed Matter Physics and Institute of Physics, Chinese Academy of Sciences, Beijing 100080, \\ China \\ ${ }^{2}$ Department of Physics and the Center of Theoretical and Computational Physics, The University of Hong Kong, Pokfulam Road, \\ Hong Kong, China
}

(Received 17 October 2006; revised manuscript received 21 December 2006; published 15 February 2007)

\begin{abstract}
We study the spin current induced spin-Hall effect that a longitudinal spin-dependent chemical potential $q V_{s=x, y, z}$ induces transverse spin conductances $G^{s s^{\prime}}$. A four-terminal system with the Rashba and Dresselhaus spin-orbit interactions (SOIs) in the scattering region is considered. By using Landauer-Büttiker formula with the aid of Green's function, various spin current induced spin-Hall conductances $G^{s s^{\prime}}$ are calculated. With the charge chemical potential $q V_{c}$ or spin chemical potential $q V_{s=x, y, z}$, there are 16 elements for the transverse conductances $G_{p}^{\mu \nu}=J_{p, \mu} / V_{\nu}$, where $\mu, \nu=x, y, z, c$. Due to the symmetry of our system, these elements are not independent. For the system with $C_{2}$ symmetry half of the elements are zero when only the Rashba SOI or the Dresselhaus SOI exists in the center region. The numerical results show that of all the conductance elements, the spin current induced spin-Hall conductances $G^{s s^{\prime}}$ are usually much greater (about one or two orders of magnitude) than the spin-Hall conductances $G^{s c}$ and the reciprocal spin-Hall conductances $G^{c s}$. So the spin current induced spin-Hall effect dominates in the present device.
\end{abstract}

DOI: 10.1103/PhysRevB.75.075324

PACS number(s): 72.25.Mk, 72.20.-i, 73.23.-b

\section{INTRODUCTION}

Recently, an interesting phenomenon, the spin-Hall effect, has been discovered in the spin-orbit interaction (SOI) system and has attracted considerable attention. In this effect, a longitudinal external bias (or named charge bias hereafter) or electric field induces a transverse spin current or spin accumulations along transverse edges. The spin-Hall effect can either be extrinsic or intrinsic. The extrinsic spin-Hall effect is due to the spin-dependent scattering ${ }^{1}$ and was found a few decades ago. On the other hand, the intrinsic spin-Hall effect is due to the SOI, predicted by Murakami et al. and Sinova et al. in a Luttinger SOI three-dimensional $p$-doped semiconductor $^{2}$ and a Rashba SOI two-dimensional (2D) electron gas, ${ }^{3}$ respectively. Since then, a great many sequent works have focused on this interesting effect. On the experimental side, two groups by Kato et ll $^{4}$ and Wunderlich et $a l .{ }^{5}$ have observed the transverse opposite spin accumulations near the two edges of their devices when the longitudinal voltage bias is added. More recently, a third group by Valenzuela and Tinkham have taken the electronic measurement of the spin-Hall effect, ${ }^{6}$ and they have observed an induced transverse voltage in a diffusive metallic conductor when a longitudinal net spin current flows through it.

Very recently, the reciprocal spin-Hall effect ${ }^{7,8}$ has been investigated, in which a transverse charge conductance is induced by the driving of a longitudinal spin-dependent bias (named spin bias hereafter). The Onsager reciprocal relation ${ }^{7}$ between the spin-Hall effect and its reciprocal phenomenon has been found, and the spin-Hall conductance is predicted to be equal to its reciprocal. So far, the spin-polarization direction considered in most of the papers on the spin-Hall effect $^{2,3}$ and its reciprocal effect ${ }^{7}$ is along the $z$-direction that is perpendicular to the $2 \mathrm{D}$ electron gas. The spin is a vector and it can also be polarized in the $x, y$, as well as in any $\hat{n}$ directions. So the direction of the output spin in the two transverse terminals (i.e., terminals 2 and 4 in Fig. 1) can be in the plane of the $2 \mathrm{D}$ electron gas, e.g., the $x$ or the $y$ direction, which has been studied by Nikolić et al. ${ }^{9,10}$ The results show that while under a longitudinal charge bias, the spin currents of the $y$ direction in the two transverse terminals are equal, i.e., they are simultaneously flowing out or flowing in (see the left inset in Fig. 1). On the other hand, in the reciprocal spin-Hall effect, the spin-polarization direction for the spin bias in the two longitudinal terminals (i.e., terminals 1 and 3 in Fig. 1) can also be in the plane of the 2D electron gas. A few theoretical works have studied this case ${ }^{11}$ and found that the charge currents in terminals 2 and 4 simultaneously flow out or in (see the left inset in Fig. 1), while the spin polarization of the spin bias is in the $y$ direction. This result is different from the usual reciprocal spin-

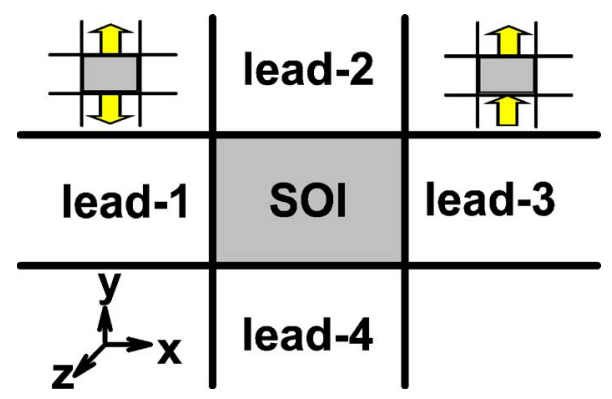

FIG. 1. (Color online) Schematic diagram for the four-terminal rectangular sample with the Rashba and Dresselhaus SOIs in the center region. The four leads are ideal without SOI. The spin bias $V_{s}$ or the charge bias $V_{c}$ is added on lead-1 and lead-3, while the induced transverse spin current $J_{p, s}$ or the charge current $J_{p, c}$ is probed in lead-2 and lead-4. The left and right insets depict the directions of the transverse spin or charge currents when the currents are flowing out $\left(G_{2}=G_{4}\right)$ and the currents are simultaneously flowing in at one terminal and out at the other one $\left(G_{2}=-G_{4}\right)$, respectively. 
TABLE I. Symmetry of the transverse spin or charge conductance for the system with the Rashba and/or Dresselhaus SOI: (a) $V_{R} \neq 0, V_{D}=0$; (b) $V_{R}=0, V_{D} \neq 0$; (c) $V_{R} \neq V_{D} \neq 0$; and (d) $V_{R}=V_{D} \neq 0$. The symbol "0" indicates the corresponding $G_{2(4)}^{\mu \nu}=0$, and the symbol " $+(-)$ " denotes $G_{2}^{\mu \nu}= \pm G_{4}^{\mu \nu}$.

\begin{tabular}{l|cccc|c|cccc} 
(a) & $V_{x}$ & $V_{y}$ & $V_{z}$ & $V_{c}$ & $(\mathrm{~b})$ & $V_{x}$ & $V_{y}$ & $V_{z}$ & $V_{c}$ \\
\hline$J_{x}$ & 0 & - & + & 0 & $J_{x}$ & 0 & - & 0 & + \\
$J_{y}$ & - & 0 & 0 & + & $J_{y}$ & - & 0 & + & 0 \\
$J_{z}$ & + & 0 & 0 & $-a_{z}$ & $J_{z}$ & 0 & + & 0 & $-_{c}$ \\
$J_{c}$ & 0 & + & $-{ }_{a}$ & 0 & $J_{c}$ & + & 0 & ${ }_{c}$ & 0 \\
\hline$(\mathrm{c})$ & $V_{x}$ & $V_{y}$ & $V_{z}$ & $V_{c}$ & $(\mathrm{~d})$ & $V_{x}$ & $V_{y}$ & $V_{z}$ & $V_{c}$ \\
\hline$J_{x}$ & - & - & + & + & $J_{x}$ & $-r_{r}$ & $-_{s}$ & $+_{o}$ & $+_{e}$ \\
$J_{y}$ & - & - & + & + & $J_{y}$ & $-{ }_{s}$ & $-r_{r}$ & $+_{\bar{o}}$ & $+_{e}$ \\
$J_{z}$ & + & + & - & - & $J_{z}$ & $+_{u}$ & $+_{u}$ & - & 0 \\
$J_{c}$ & + & + & - & - & $J_{c}$ & $+_{v}$ & $+_{\bar{v}}$ & 0 & - \\
\hline \hline
\end{tabular}

Hall effect with the $z$-direction spin bias, in which the charge current flows in at one transverse terminal and out at the other one (see the right inset in Fig. 1).

To explore the vector nature of the spin-Hall effect, we set the longitudinal bias to be spin-dependent and examine the transverse spin conductance; that is, the transverse spin currents are induced by the longitudinal spin bias, which is named the spin current induced spin-Hall effect hereafter. So far, most of the studies focus on the spin-Hall effect and its reciprocal effect, and less attention has been paid to the spin current induced spin-Hall effect. It is the purpose of this paper to fill this gap. We study the spin current induced spinHall effect in a finite mesoscopic system by using the Landauer-Büttiker formula with the aid of Green's function. We consider a four-terminal ballistic 2D rectangular region and the center rectangular region as having the Rashba and Dresselhaus SOIs (as shown in Fig. 1). A spin bias $V_{s}(s=x$, $y$, and $z$ ) is added at the longitudinal terminals 1 and $3 .{ }^{12}$ The spin bias can be provided from the device of the spin cell, which has been suggested by some recent theoretical works ${ }^{13}$ and has also been realized in a few experimental works. ${ }^{14}$ Under the spin bias $V_{s}$, the chemical potentials $\mu$ for the spin-up and spin-down electrons in terminals 1 and 3 are split, and $\mu_{1, s \uparrow}=-\mu_{1, s \downarrow}=-\mu_{3, s \uparrow}=\mu_{3, s \downarrow}=e V / 2$, where $s=x, y$, and $z$ represents the spin-polarization direction. For comparison, we also consider the charge bias $V_{c}$ on the longitudinal terminals 1 and 3 ; in this case, $\mu_{1, s \uparrow}=\mu_{1, s \downarrow}=-\mu_{3, s \uparrow}=-\mu_{3, s \downarrow}$ $=e V / 2 .{ }^{15}$ The transverse two terminals 2 and 4 act as the measuring terminals, and their chemical potentials (or the terminal bias) are set to zero. Due to the SOI, the spin bias $V_{s}$ or the charge bias $V_{c}$ can induce transverse spin-Hall currents $J_{s}$ as well as the charge-Hall current $J_{c}$, which will be investigated in this paper. For this purpose, we use four component vectors $\mathbf{J}=\left(J_{x}, J_{y}, J_{z}, J_{c}\right)$ and $\mathbf{V}=\left(V_{x}, V_{y}, V_{z}, V_{c}\right)$ to represent the spin (charge)-Hall current and spin (charge) bias, respectively. In the small bias limit, the relationship between $\mathbf{J}$ and $\mathbf{V}$ can be characterized by a $4 \times 4$ matrix $G^{\mu \nu}(\mu, \nu$ $\in\{x, y, z, c\})$.
We have studied the relations among these matrix elements from the symmetry point of view. Four cases have been discussed: (1) only the Rashba SOI is present, (2) only the Dresselhaus SOI is present, (3) both the Rashba and Dresselhaus SOIs are present, and (4) the Rashba and Dresselhaus SOIs have the same interaction strength. The results are summarized in Table I. All elements are found to have the property $\left|G_{2}^{\mu \nu}\right|=\left|G_{4}^{\mu \nu}\right|$, where $G_{p}^{\mu \nu}=J_{p, \mu} / V_{\nu}$ with $p$ $=2,4 .{ }^{16}$ This means that the absolute value $\left|J_{2, \mu}\right|$ of the spin $(\mu=x, y, z)$ current or the charge $(\mu=c)$ current in terminal 2 is equal to $\left|J_{4, \mu}\right|$ in terminal 4 regardless of longitudinal driving bias $V_{\nu}$. From Table I, we see that if only the Rashba SOI or the Dresselhaus SOI is present in the center region, half of the matrix elements of the transverse conductances $G^{\mu \nu}$ are zero. We have also numerically studied the behavior of these nonzero spin matrix elements and found that the spin current induced spin-Hall conductances are much larger (about one or two orders of magnitude) than the spin-Hall conductance as well as its reciprocal one.

\section{HAMILTONIAN AND SOLUTION}

The system that we considered is sketched in Fig. 1. The central gray region is the semiconductor sample in which the SOIs of Rashba or/and Dresselhaus are present. The sample is connected to four ideal non-SOI leads. The Hamiltonian of the central region is given by $H_{0}=p^{2} / 2 m^{*}+V(x, y)+(\alpha / \hbar)$ $\times\left(\sigma_{x} p_{y}-\sigma_{y} p_{x}\right)-(\beta / \hbar)\left(\sigma_{x} p_{x}-\sigma_{y} p_{y}\right)$, where $\alpha$ and $\beta$ are the coefficients of the Rashba and Dresselhaus SOIs and $V(x, y)$ is the hard wall confining potential. In the tight-binding representation, the total Hamiltonian can be written as ${ }^{17-19}$

$$
\begin{aligned}
H= & \sum_{i}\left[-t a_{i}^{\dagger} a_{i+\delta x}-t a_{i}^{\dagger} a_{i+\delta y}+i a_{i}^{\dagger}\left(V_{R} \sigma_{y}+V_{D} \sigma_{x}\right) a_{i+\delta x}\right. \\
& \left.-i a_{i}^{\dagger}\left(V_{R} \sigma_{x}+V_{D} \sigma_{y}\right) a_{i+\delta y}\right]+ \text { H.c. },
\end{aligned}
$$

where $a_{i}^{\dagger}=\left(a_{i \uparrow}^{\dagger}, a_{i \downarrow}^{\dagger}\right), i$ is the lattice site in the central region and the leads, $t=\hbar^{2} / 2 m^{*} a^{2}$ is the hopping matrix element 
with the lattice constant $a, \delta x$ and $\delta y$ are the unit vectors along the $x$ and $y$ directions, and $\sigma_{x}, \sigma_{y}$ are Pauli matrices. Here, $V_{R}=\alpha / 2 a$ and $V_{D}=\beta / 2 a$ represent the strength of the Rashba and Dresselhaus SOIs, respectively. $V_{R}$ and $V_{D}$ are nonzero only in the central gray region.

Since there is no SOI in the leads, the particle current $J_{p, s \sigma}$ in the lead- $p(p=2,4)$ with spin at the $\pm s$ direction $(\sigma=\uparrow$ or $\downarrow$ stands for the $+s$ or $-s$ direction with $s=x, y, z)$ due to the longitudinal spin or charge bias $V_{\mu}$ can be obtained from the Landauer-Büttiker formula ${ }^{20} J_{p, s \sigma}$ $=(1 / h) \Sigma_{q, \sigma^{\prime}} T_{p s \sigma, q s^{\prime} \sigma^{\prime}}\left(\mu_{p, s \sigma^{-}}-\mu_{q, s^{\prime} \sigma^{\prime}}\right)$, where $\mu_{p, s \sigma}$ and $\mu_{q, s^{\prime} \sigma^{\prime}}$ are the spin-dependent or spin independent chemical potential related to the bias $V_{\mu}$, which has been detailed in the the Introduction. $T_{q s^{\prime} \sigma^{\prime}, p s \sigma}$ is the transmission coefficient from the lead- $p$ with spin $s \sigma$ to the lead- $q$ with spin $s^{\prime} \sigma^{\prime}$. Note that the spin indices $\uparrow$ and $\downarrow$ in the Hamiltonian ((1)) represent the spin pointing to the $+z$ and $-z$ directions, and not pointing to the $\pm x$ and $\pm y$ directions. So in order to calculate the transmission coefficient $T_{q s^{\prime} \sigma^{\prime}, p s \sigma}$ for $s \neq z$ and/or $s^{\prime} \neq z$, we need to rotate the $z$-axis in the spin space in the lead- $p$ (lead- $q$ ) to the $s$-direction ( $s^{\prime}$-direction) by taking a unitary transformation:

$$
\left(\begin{array}{c}
a_{i \uparrow}^{\prime} \\
a_{i \downarrow}^{\prime}
\end{array}\right)=\mathbf{U}_{s\left(s^{\prime}\right)}^{\dagger}\left(\begin{array}{c}
a_{i \uparrow} \\
a_{i \downarrow}
\end{array}\right),
$$

where $i$ is the lattice site in the lead- $p(q)$. The operator $a_{i \sigma}$ in the center region and in the other two leads does not change. The unitary matrix $\mathbf{U}_{s}$ in Eq. (2) is

$$
\mathbf{U}_{s}=\left(\begin{array}{cc}
\cos \frac{\theta}{2} & e^{-i \phi} \sin \frac{\theta}{2} \\
e^{i \phi} \sin \frac{\theta}{2} & -\cos \frac{\theta}{2}
\end{array}\right),
$$

where $(\theta, \phi)$ is the directional angle of the $s$-direction and $\mathbf{U}_{s}^{\dagger} \sigma_{s} \mathbf{U}_{s}=\sigma_{z}$. Under this unitary transformation, the Hamiltonian of the leads does not vary because the leads' Hamiltonian does not include the Pauli matrix; only the Hamiltonian that describes the coupling between the leads and the center region changes. After the unitary transformation, the $z$-axis of the spin in the lead- $p(q)$ is in the $s\left(s^{\prime}\right)$-direction; then the transmission coefficient $T_{q s^{\prime} \sigma^{\prime}, p s \sigma}$ can be easily obtained as ${ }^{20}$

$$
T_{q s^{\prime} \sigma^{\prime}, p s \sigma}=\operatorname{Tr}\left[\boldsymbol{\Gamma}_{q, s^{\prime} \sigma^{\prime}} \mathbf{G}^{r} \boldsymbol{\Gamma}_{p, s \sigma} \mathbf{G}^{a}\right],
$$

where the linewidth function $\boldsymbol{\Gamma}_{p, s \sigma}=i\left(\boldsymbol{\Sigma}_{p, s \sigma}^{r}-\boldsymbol{\Sigma}_{p, s \sigma}^{r \dagger}\right)=\boldsymbol{\Gamma}_{p}$ $\otimes \mathbf{U}_{s} \mathbf{S}_{\sigma} \mathbf{U}_{s}^{\dagger}$, with the retarded self-energy $\quad \mathbf{\Sigma}_{p, s \sigma}^{r}=\mathbf{\Sigma}_{p}^{r}$ $\otimes \mathbf{U}_{s} \mathbf{S}_{\sigma} \mathbf{U}_{s}^{\dagger}$ where $\boldsymbol{\Gamma}_{p}$ and $\boldsymbol{\Sigma}_{p}^{r}$ are, respectively, the linewidth function and the self-energy of the lead- $p$ for no-spin-index lattice system. Here, the matrix $\mathbf{S}_{\sigma}$ is

$$
\mathbf{S}_{\uparrow}=\left(\begin{array}{ll}
1 & 0 \\
0 & 0
\end{array}\right), \quad \mathbf{S}_{\downarrow}=\left(\begin{array}{ll}
0 & 0 \\
0 & 1
\end{array}\right) .
$$

The retarded and advanced Green's functions $\mathbf{G}^{r, a}$ in Eq. (4) can be calculated from ${ }^{20} \mathbf{G}^{r}=\left[\mathbf{G}^{a}\right]^{\dagger}=\left\{E_{F} \mathbf{I}-\mathbf{H}_{0}-\Sigma_{p, \sigma} \mathbf{\Sigma}_{p, s \sigma}^{r}\right\}^{-1}$, with the unit matrix I. Since $\Sigma_{\sigma} \boldsymbol{\Sigma}_{p, s \sigma}^{r}=\mathbf{\Sigma}_{p}^{r} \otimes \Sigma_{\sigma}\left(\mathbf{U}_{s} \mathbf{S}_{\sigma} \mathbf{U}_{s}^{\dagger}\right)$ $=\boldsymbol{\Sigma}_{p}^{r} \otimes \mathbf{I}$ that is independent of the spin-direction index $s$, the
Green's functions $\mathbf{G}^{r, a}$ are also independent of the spindirection index. This, in turn, indicates that the Green's functions $\mathbf{G}^{r, a}$ remain unchanged in the above unitary transformation, in which the spin axis in the leads is rotated.

Once the particle currents $J_{p, s \sigma}(p=2,4)$ are obtained, the spin current $J_{p, s}$ and the charge current $J_{p, c}$ can be obtained straightforwardly: $J_{p, c}=e\left\{J_{p, s \uparrow}+J_{p, s \downarrow}\right\}$ and $J_{p, s}=(\hbar / 2)\left\{J_{p, s \uparrow}\right.$ $\left.-J_{p, s \downarrow}\right\}$, where $s=x, y, z$. In fact, it is easy to show that the charge current $J_{p, c}$ is independent of the spin-direction index $s$ of the lead- $p$ (i.e., $J_{p, x \uparrow}+J_{p, x \downarrow}=J_{p, y \uparrow}+J_{p, y \downarrow}=J_{p, z \uparrow}+J_{p, z \downarrow}$ $\left.=J_{p, \hat{n} \uparrow}+J_{p, \hat{n} \downarrow}\right)$, so the subscript $s$ is neglected from now on. Then, from the current $\mathbf{J}_{\mathbf{p}}=\left(J_{p, x}, J_{p, y}, J_{p, z}, J_{p, c}\right)$ and its driving bias $\mathbf{V}=\left(V_{x}, V_{y}, V_{z}, V_{c}\right)$, we get all the 16 conductance matrix elements $G_{p}^{\mu \nu}=J_{p, \mu} / V_{\nu}(\mu, \nu \in c, x, y, z) .{ }^{16}$ They are the charge-Hall conductance $G_{p}^{c c}$ describing the transverse charge current $J_{p, c}$ induced by the longitudinal charge bias $V_{c}$, the spin-Hall conductance $G_{p}^{s c}$ for the transverse spin current $J_{p, s}$ induced by the longitudinal charge bias $V_{c}$, the reciprocal spin-Hall conductance $G_{p}^{c s}$ representing the transverse charge current $J_{p, c}$ induced by the longitudinal spin bias $V_{s}$, and the spin-Hall conductance $G_{p}^{s{ }^{\prime}}$ induced by the spin current describing the transverse spin current $J_{p, s}$ induced by the longitudinal spin bias $V_{s}$.

\section{RELATIONS DUE TO SYMMETRY}

We now study the relations among the 16 conductance matrix elements $G_{p}^{\mu \nu}$ considering the symmetry of the device. First, our system satisfies the time-reversal symmetry, ${ }^{21}$ so the transmission coefficients satisfy the relation $T_{p s \sigma, q s^{\prime} \sigma^{\prime}}$ $=T_{q s^{\prime} \bar{\sigma}, p s \bar{\sigma}}$, which determines the properties of the transverse conductances. Second, if the shape of the device has the geometrical symmetry, the results can be greatly simplified and many matrix elements are zero. In the following, we consider the rectangular center region that has $C_{2}$ symmetry.

For the rectangular sample, the shape of the device [i.e., the confining potential $V(x, y)]$ is invariant under the rotation transformation $C_{2}$ by rotating an angle $\pi$ around the $x, y, z$-axis at the center point. However, the SOI part of the Hamiltonian is varied under the space rotation transformation $C_{2}$. In order to keep the invariance of the total Hamiltonian $H$, we construct the unitary transformation $U=U_{0}$ $\otimes U_{s}$, where $U_{0}$ and $U_{s}$ are the rotation transformations in the real space and in the spin space, respectively. In the following, we list all of the unitary transformations $U$, under which the total Hamiltonian $H$ is invariant (i.e., $U^{\dagger} H U=H$ ). We then derive the relations among the transverse conductance matrix elements from the symmetry. We consider four cases of SOI: (1) with the Rashba SOI only, (2) with the Dresselhaus SOI only, (3) with both the Rashba and Dresselhaus SOIs, and (4) similar to (3) but the strengths of the Rashba and Dresselhaus SOIs are equal. To illustrate the derivation, we shall discuss the case of the Rashba SOI case in detail. For the other three cases, similar discussions apply and we only give the results.

First, we study the case with only the Rashba SOI using the following transformations.

(i) $U_{1}=C_{2 z} \otimes \exp \left[-i(\pi / 2) \sigma_{z}\right]$, which performs the trans- 
formation $x \rightarrow-x, y \rightarrow-y, z \rightarrow z$ and $\sigma_{x} \rightarrow-\sigma_{x}, \sigma_{y} \rightarrow-\sigma_{y}$, $\sigma_{z} \rightarrow \sigma_{z}$. Under this transformation $U_{1}$, the system (including both of the real space and the spin space) is rotated by an angle $\pi$ around the $z$-axis, which leads $J_{2, x \sigma} \rightarrow J_{4, x \bar{\sigma}}$ and $\mu_{1, x \sigma} \rightarrow \mu_{3, x \bar{\sigma}}$, so the spin current $J_{2, x}=(\hbar / 2)\left\{J_{2, x \uparrow}-J_{2, x \downarrow}\right\}$ $\rightarrow(\hbar / 2)\left\{J_{4, x \downarrow}-J_{4, x \uparrow}\right\}=-J_{4, x}$, and the spin chemical potential $V_{x}$ remains unchanged because the charge chemical potential $V_{c}$ and the $\sigma_{x}$ change signs due to the rotations in the real space and in the spin space, respectively. Similarly, the transformation for the others $J_{2, \mu}$ and $V_{\mu}$ can also be deduced as

$$
\begin{aligned}
& J_{2, c} \rightarrow J_{4, c}, \quad V_{c} \rightarrow-V_{c}, \\
& J_{2, x} \rightarrow-J_{4, x}, \quad V_{x} \rightarrow V_{x}, \\
& J_{2, y} \rightarrow-J_{4, y}, \quad V_{y} \rightarrow V_{y}, \\
& J_{2, z} \rightarrow J_{4, z}, \quad V_{z} \rightarrow-V_{z} .
\end{aligned}
$$

Then, the relations among the matrix elements of the transverse conductance can be obtained straightforwardly. For example, $G_{2}^{x x}=J_{2, x} / V_{x}=-J_{4, x} / V_{x}=-G_{4}^{x x}$, etc.

(ii) $U_{2}=C_{2 y} \otimes \exp \left[-i(\pi / 2) \sigma_{x}\right]$, which performs the transformation $x \rightarrow-x, y \rightarrow y, z \rightarrow-z$ and $\sigma_{x} \rightarrow \sigma_{x}, \sigma_{y} \rightarrow-\sigma_{y}, \sigma_{z}$ $\rightarrow-\sigma_{z}$. This transformation $U_{2}$ is equivalent to rotating the real space by $\pi$ around the $y$-axis and to rotating the spin by $\pi$ around the $x$-axis. Under the unitary transformation $U_{2}$, $J_{p, \mu}(p=2,4)$ and $V_{\mu}$ are transformed into

$$
\begin{aligned}
& J_{2(4), c} \rightarrow J_{2(4), c}, \quad V_{c} \rightarrow-V_{c}, \\
& J_{2(4), x} \rightarrow J_{2(4), x}, \quad V_{x} \rightarrow-V_{x}, \\
& J_{2(4), y} \rightarrow-J_{2(4), y}, \quad V_{y} \rightarrow V_{y}, \\
& J_{2(4), z} \rightarrow-J_{2(4), z}, \quad V_{z} \rightarrow V_{z} .
\end{aligned}
$$

Then, $G_{p}^{c c}=J_{p, c} / V_{c}=J_{p, c} /\left(-V_{c}\right)=-G_{p}^{c c}$, so $G_{p}^{c c}=0(p=2,4)$. In fact, from $U_{2}^{\dagger} H U_{2}=H$, we obtain the fact that eight matrix elements of the transverse conductance are zero, $G_{p}^{c c}=G_{p}^{c x}$ $=G_{p}^{x c}=G_{p}^{x x}=G_{p}^{y y}=G_{p}^{y z}=G_{p}^{z y}=G_{p}^{z z}=0 \quad(p=2,4)$.

(iii) $U_{3}=C_{2 x} \otimes \exp \left[-i(\pi / 2) \sigma_{y}\right]$, which performs the transformation $x \rightarrow x, y \rightarrow-y, z \rightarrow-z$ and $\sigma_{x} \rightarrow-\sigma_{x}, \sigma_{y} \rightarrow \sigma_{y}$, $\sigma_{z} \rightarrow-\sigma_{z}$. This transformation $U_{3}$ rotates the real space by $\pi$ around the $x$-axis and the spin by $\pi$ around the $y$-axis. Under this transformation, $J_{p, \mu}(p=2,4)$ and $V_{\mu}$ are transformed into

$$
\begin{gathered}
J_{2, c} \rightarrow J_{4, c}, \quad V_{c} \rightarrow V_{c}, \\
J_{2, x} \rightarrow-J_{4, x}, \quad V_{x} \rightarrow-V_{x}, \\
J_{2, y} \rightarrow J_{4, y}, \quad V_{y} \rightarrow V_{y}, \\
J_{2, z} \rightarrow-J_{4, z}, \quad V_{z} \rightarrow-V_{z} .
\end{gathered}
$$

It is worth mentioning that the above three unitary transformations are not independent of each other, and we have $U_{3} U_{1}=U_{2}$. So Eq. (7) can be obtained from Eqs. (6) and (8).
Combining Eqs. ${ }^{6-8}$, the relations among the matrix elements of the transverse conductances $G_{p}^{\mu \nu}$ are obtained and summarized in Table I(a). The 16 conductance matrix elements are arranged in the matrix form, in which the columns (labeled by $J_{\mu}$ ) denote the measured transverse conductances (from lead 2 or 4) and the rows are the longitudinal bias $V_{\nu}$ in lead 1 or 3 . The zero conductance matrix elements are indicated by the symbol " 0 ." The symbol "+ (-)" denotes the nonzero conductance matrix elements $G_{p}^{\mu \nu}$, which have the same (opposite) signs for lead-2 and lead-4, i.e., $G_{2}^{\mu \nu}$ $= \pm G_{4}^{\mu \nu}$. For some pair of conductance matrix elements, they may have the same value or they may differ in sign, e.g., $G_{p}^{c z}=G_{p}^{z c}, p=2,4$, which is marked by the letter symbols in Table I. Here, different symbols present different conductance values, and the symbol $a$ (or $\bar{a}$ ) is for $G_{2}^{\mu \nu}=a$ (or $\left.G_{2}^{\mu \nu}=-a\right)$.

To examine the system with the Dresselhaus SOI only, we need only change $\sigma_{x}$ to $\sigma_{y}$ and $\sigma_{y}$ to $\sigma_{x}$ in the above Hamiltonian with the Rashba SOI and unitary transformations. As a result, the above three transformations are changed to $U_{1}$ $=C_{2 z} \otimes \exp \left[-i(\pi / 2) \sigma_{z}\right], U_{2}=C_{2 y} \otimes \exp \left[-i(\pi / 2) \sigma_{y}\right]$, and $U_{3}$ $=C_{2 x} \otimes \exp \left[-i(\pi / 2) \sigma_{x}\right]$. Under these transformations, we find all the symmetry relations among the 16 conductances and the results are listed in Table I(b). In the case that we have both the Rashba and Dresselhaus SOIs $\left(V_{R} \neq 0\right.$ and $V_{D} \neq 0$ ), only the transformation $U_{1}$ can keep the Hamiltonian invariant, and it leads to the symmetry relations shown in Table I(c). In the case of $V_{R}=V_{D} \neq 0$, except $U_{1}$, there is an additional symmetry $U_{4}: \mathbf{I} \otimes\left(\sigma_{x(y)} \rightarrow \sigma_{y(x)}, \sigma_{z}\right.$ $\rightarrow \sigma_{z}$ ), which leads to the symmetry between the $s_{x}$ and $s_{y}$, and the relations of the conductance elements $G_{p}^{\mu \nu}$ are expressed in Table $\mathrm{I}(\mathrm{d})$. In fact, $U_{4}$ only takes the transformation in the spin space; so the symmetry $U_{4}$ always exists and $G_{p}^{z c}=G_{p}^{c z}=0$ for any confining potential $V(x, y)$ (not requiring the rectangular sample).

From these symmetry relations, our conclusions are as follows. (i) All the conductance matrix elements are found to obey the relation $\left|G_{2}^{\mu \nu}\right|=\left|G_{4}^{\mu \nu}\right|$, i.e., the absolute value of $\left|J_{2, \mu}\right|$ of the spin $(\mu=x, y, z)$ or the charge $(\mu=c)$ current in terminal 2 is equal to the absolute value of $\left|J_{4, \mu}\right|$ in terminal 4. Furthermore, $G_{2}^{\mu \nu}=-G_{4}^{\mu \nu}$ for the eight block-diagonal elements in Table I, and $G_{2}^{\mu \nu}=G_{4}^{\mu \nu}$ for the eight non-blockdiagonal elements. (ii) Exchanging $\sigma_{x}$ and $\sigma_{y}$, we find the system with the Rashba SOI to be the same as that with the Dresselhaus SOI, which can be seen from Tables I(a) and (b). (iii) If only the Rashba SOI or the Dresselhaus SOI is present in the center region, half of the matrix elements $G^{\mu \nu}$ are zero. $^{22}$ (vi) For the usual spin-Hall effect or its reciprocal effect (in which the spin is polarized along the $z$-direction), the transverse spin current or charge current is conserved (i.e., $\left.G_{2}^{z c(c z)}=-G_{4}^{z c(c z)}\right)$. Furthermore, $G^{z c}$ and $G^{c z}$ satisfy the Onsager relation $G_{2(4)}^{z c}=G_{2(4)}^{c z}{ }^{7,23}$ For $G^{c y}$ and $G^{y c}$, as well the non-block-diagonal elements in Table I, they either flow in or out of terminals 2 and 4 (i.e., $G_{2}=G_{4}$ ), and they do not satisfy the Onsager relation because that Onsager relation requires that the currents in the two transverse terminals 2 and 4 must be $J_{2}=-J_{4}$. 


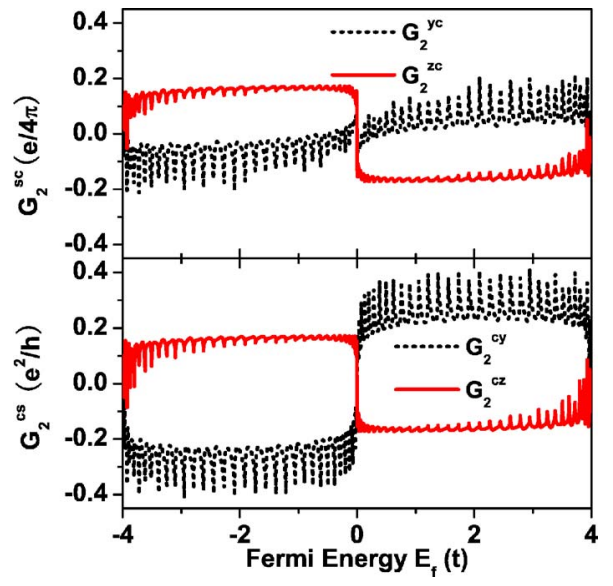

FIG. 2. (Color online) The spin-Hall conductances $G^{s c}$ and the reciprocal spin-Hall conductances $G^{c s}$ vs Fermi energy $E_{F}$, with the parameters $L=34 a$ and $V_{R}=0.03 t$.

\section{NUMERICAL RESULTS AND DISCUSSION}

In the following, we numerically study the nonzero conductance matrix elements $G_{p}^{\mu \nu}$, and we mainly focus on the spin-Hall conductance induced by the spin current $G_{p}^{s s^{\prime}}$ $\left(s, s^{\prime} \in x, y, z\right)$. For simplicity, only the case of the Rashba SOI is shown since the results for the other three cases have similar physics. In the numerical calculation, we consider the square scattering center region for convenience. However, notice that the symmetry relation in Table I is valid for the rectangular scattering center region. In the calculation, the electron effective mass $m^{*}$ is set to $0.05 m_{e},{ }^{24,25}$ and the Fermi energy $E_{F}=-3.8 t$ (in Figs. 4 and 5), which is near the band bottom $-4 t$, with $t=1$ as an energy unit and the corresponding lattice constant $a \approx 2.6 \mathrm{~nm} .{ }^{9,10}$ The size of the center region $L$ is chosen in the same order as the spin precession length $L_{\mathrm{SO}}\left(L_{\mathrm{SO}} \equiv \pi a t / 2 V_{R}\right)$ over the precessing angle $\pi$. If taking $V_{R}=0.03 t$ (corresponding to $\alpha \approx 1 \times 10^{-11} \mathrm{eV} \mathrm{m}$ ), $L_{\mathrm{SO}} \approx 50 a$.

First, Fig. 2 shows the spin-Hall conductance $G^{z c}$, the reciprocal spin-Hall conductance $G^{c z}$, the in-plane spin-Hall conductance $G^{y c}$, and its reciprocal conductance $G^{c y}$. We see that they are odd functions of the Fermi energy $E_{F}$ and vanish at the half-filled band with $E_{F}=0$. The behaviors of the spin-Hall conductance $G^{z c}$ and $G^{y c}$ are similar to those in the previous works done by Nikolić et al. ${ }^{9,10}$ From Fig. 2, we can find that the out-of-plane matrix elements $G^{z c}=G^{c z}$, which satisfies the Onsager relation. ${ }^{7}$ On the other hand, the in-plane matrix elements $G^{y c}$ and $G^{c y}$ do not obey the Onsager relation because $G^{y c}$ and $G^{c y}$ simultaneously flow out of or into the two transverse terminals 2 and 4 (as shown in the left inset of Fig. 1), and they do not satisfy the corresponding condition. $G^{y c}$ is smaller than $G^{c y}$, and both of them strongly depend on the Fermi energy.

Second, we examine the spin-Hall conductances induced by the spin current $G^{s s^{\prime}}\left(s, s^{\prime} \in x, y, z\right)$. Figure 3 shows the four nonzero matrix elements $G^{s s^{\prime}}$ versus the Fermi energy $E_{F}$. Different from $G^{s c}$ and $G^{c s}$, the spin current induced spin-Hall conductances $G^{s s^{\prime}}$ are even functions of $E_{F}$, and

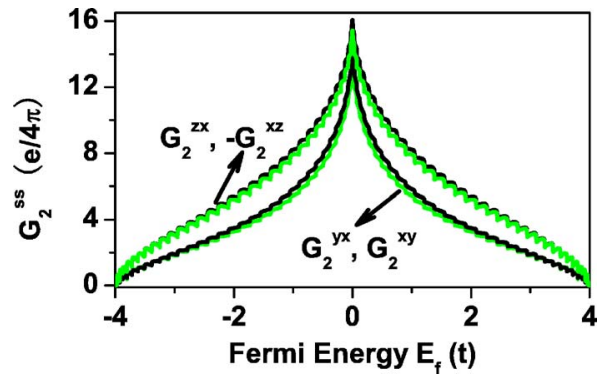

FIG. 3. (Color online) The nonzero spin current induced spinHall conductance elements $G_{2}^{x y}$ (black), $G_{2}^{y x}$ (green or gray), $G_{2}^{z x}$ (black), and $-G_{2}^{x z}$ (green or gray) vs Fermi energy $E_{F}$. The parameters are $L=34 a$ and $V_{R}=0.03 t$.

$\left|G^{s s^{\prime}}\right|$ reaches the largest value at the half-filled band with $E_{F}=0$. In particular, the spin current induced spin-Hall conductances $G^{s s^{\prime}}$ are much larger (at least one order of magnitude larger) than $G^{s c}$ or $G^{c s}$ (see Figs. 2 and 3). This means that the spin current induced spin-Hall effect is the dominating effect in the SOI system. The spin current induced spinHall conductances $G^{s s^{\prime}}$ do not obey the Onsager relations; in general, $G^{x y} \neq G^{y x}$ and $G^{x z} \neq G^{z x}$. This is because the present device is not simply a four-terminal system, but more likely an eight-terminal system with the index of both the terminal and the spin. Anothr reason is that the circuit of the Onsager relations requires that the boundary condition of the transverse terminals 2 and 4 be $J_{2}=-J_{4}$, i.e., in the external circuit, terminals 2 and 4 and terminals 1 and 3 are connected, respectively. However, in the present system, the current $G_{2}^{\mu \nu} \neq-G_{4}^{\mu \nu}$ for the non-block-diagonal elements. On the other hand, for the small $V_{R}$ (e.g., $\left.V_{R}<0.08 t\right),\left|G^{x y}\right|$ and $\left|G^{x z}\right|$ are still approximately equal to $\left|G^{y x}\right|$ and $\left|G^{z x}\right|$, respectively.

The spin current induced spin-Hall conductances $G^{s s^{\prime}}$ versus the sample size $L$ and the Rashba SOI strength $V_{R}$ are plotted in Figs. 4 and 5, respectively. With the increasing size of the center region $L$, the conductances $G^{y x}$ and $G^{x y}$ are greatly enhanced (see Fig. 4) because the transverse terminals 2 and 4 are much wider at large $L$. However, at a few special sizes $L$ (e.g., $L=26,46$ for $V_{R}=0.06 t$ ), $G^{x y}$ and $G^{y x}$ are anomalous, in which $G^{x y}$ and $G^{y x}$ are minimum. This is because a subband passes the Fermi energy $E_{F}$. The conductances $G^{z x}$ and $G^{x z}$ have similar behaviors with $G^{y x}$ and $G^{x y}$ while in the small $V_{R}$ region, i.e., the corresponding spin precession length $L_{\mathrm{SO}}=\pi a t / 2 V_{R}>L$, but for the large $V_{R}$ case (i.e., $\left.L_{\mathrm{SO}}<L\right), G^{z x}$ and $G^{x z}$ are not regular with the size $L$. In the present spin-Hall device, the quantum states are extended. ${ }^{26}$

On the other hand, with the increasing Rashba SOI strength $V_{R}$, the conductances $G^{s s^{\prime}}$ are oscillatory (see Fig. 5). For the larger $V_{R}, G^{s s^{\prime}}$ shows a chaotic behavior. Furthermore, at the large $V_{R},\left|G^{x y}\right|$ and $\left|G^{x z}\right|$ are not equal to $\left|G^{y x}\right|$ and $\left|G^{z x}\right|$, i.e., the Onsager relations for the spin current induced spin-Hall conductances $G^{s s^{\prime}}$ are completely violated.

At last, we investigate how the spin current induced spinHall conductances $G^{s s^{\prime}}$ are affected by the disorders. In the previous calculation, the on-site energies $\epsilon_{i}$ (i.e., in the term $\left.\sum_{i} \epsilon_{i} a_{i}^{\dagger} a_{i}\right)$ in Eq. (1) are taken to be zero when there are no 


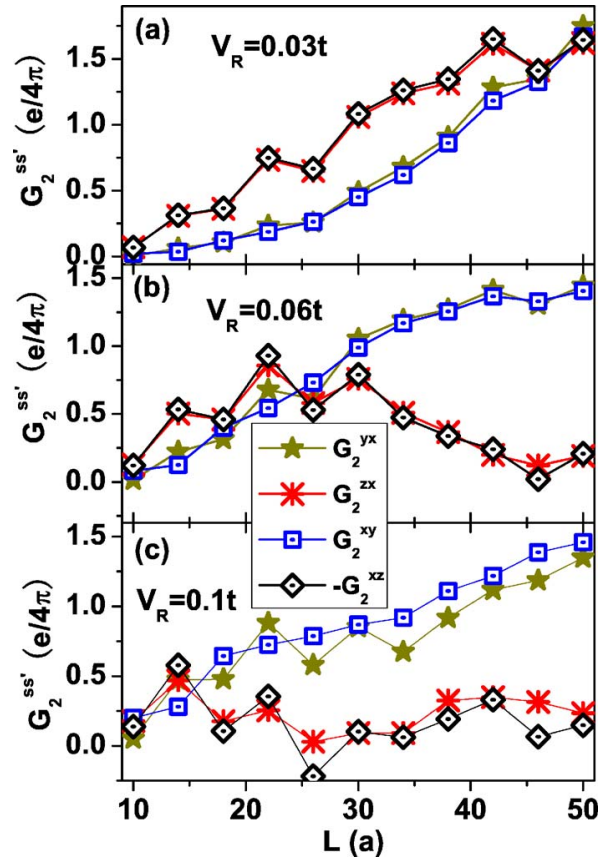

FIG. 4. (Color online) The spin current induced spin-Hall conductances $G^{s s^{\prime}}$ vs sample size $L$ for different SOI strengths: (a) $V_{R}=0.03$, (b) $V_{R}=0.06$, and (c) $V_{R}=0.1$. The curves of $G_{2}^{x y}$ and $G_{2}^{y x}$ and $-G_{2}^{x z}$ and $G_{2}^{z x}$ are nearly coincident for the small $V_{R}=0.03$ and 0.06. The Fermi energy is $E_{F}=-3.8 t$.

disorders. To consider the effect of disorder, random on-site potentials $\epsilon_{i}$ in the center region are added with a uniform distribution $[-W / 2, W / 2]$ with disorder strength $W$. The conductance is obtained by averaging over up to 1000 disorder configurations. Figure 6 shows the normalized conductances $G_{\text {ratio }}^{s s^{\prime}}\left[G_{\text {ratio }}^{s s^{\prime}} \equiv G^{s s^{\prime}}(W \neq 0) / G^{s s^{\prime}}(W=0)\right]$ versus the disorder strength $W$ for the different sizes $L$. From Fig. 6, we can see that the spin current induced spin-Hall conductances $G^{s s^{\prime}}$ decrease with the increasing disorder strength $W$, but $G^{s s^{\prime}}$ keep the large value while $W<t$. This behavior is similar to the spin-Hall conductances which have been investigated recently. ${ }^{27}$ Hence, in the dirty case, the spin current induced spin-Hall effect is still dominant in the finite 2D SOI system. In addition, for the different sizes of the device, the relation

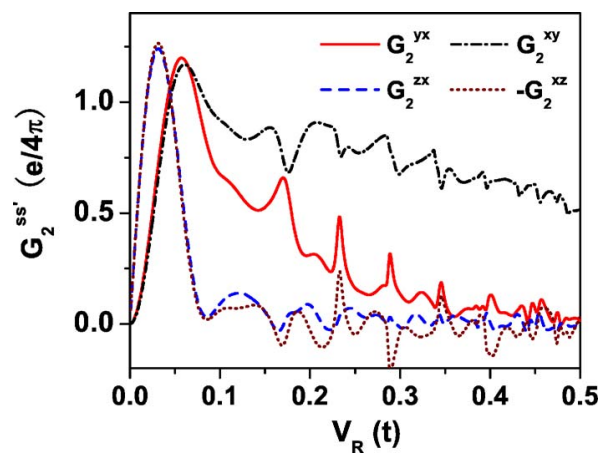

FIG. 5. (Color online) The spin current induced spin-Hall conductances $G^{s s^{\prime}}$ vs the Rashba SOI strength $V_{R}$, with the parameters $L=34 a$ and $E_{F}=-3.8 t$.

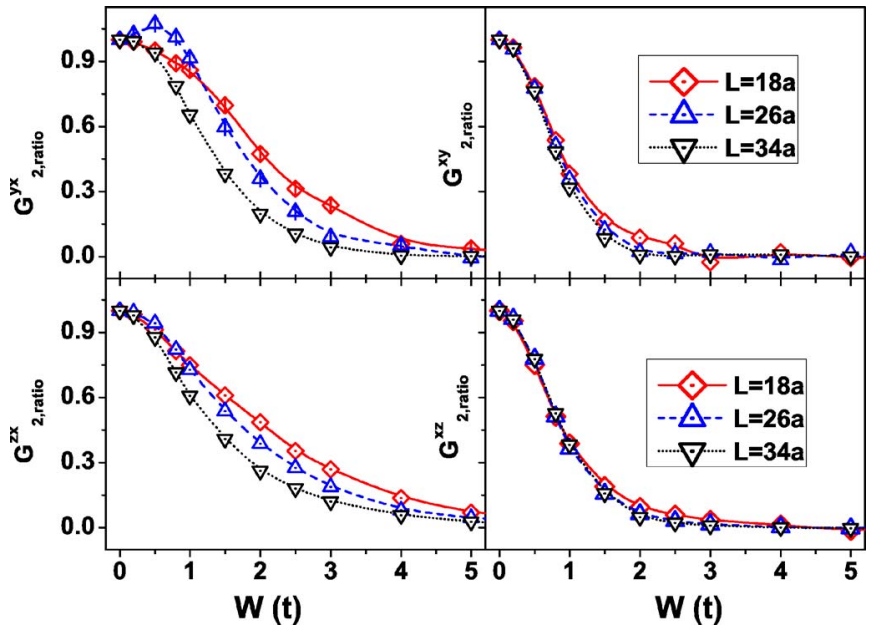

FIG. 6. (Color online) The normalized conductances $G_{\text {ratio }}^{s \prime^{\prime}}$ $\left[G_{\text {ratio }}^{s s^{\prime}}=G^{s s^{\prime}}(W \neq 0) / G^{s s^{\prime}}(W=0)\right]$ vs the disorder strength $W$ with the different sizes $L=18 a, 26 a$, and $34 a$. The other parameters are $E_{F}=-3.8 t$ and $V_{R}=0.03$.

of $G_{\text {ratio }}^{x y}$ and $G_{\text {ratio }}^{x z}$ versus $W$ almost remain the same. On the other hand, $G_{\text {ratio }}^{y x}$ and $G_{\text {ratio }}^{z x}$ can keep larger value in the system with a small size than the system with a big size for fixed disorder strength $W$.

\section{SUMMARY}

The spin current induced spin-Hall effect is investigated in a four-terminal system with the center region having the spin-orbit interaction (SOI). Because of the vector nature of spin, the charge and three spin components form a $4 \times 4$ $=16$ transverse conductance matrix whose matrix elements include the spin current induced spin-Hall conductances $G^{s s^{\prime}}$ $\left(s, s^{\prime} \in x, y, z\right)$, the spin-Hall conductances $G^{s c}$, the reciprocal spin-Hall conductances $G^{c s}$, and the charge-Hall conductances $G^{c c}$. Of these matrix elements, we found that in general, $G^{s s^{\prime}}$ are much larger (about one or two orders) than the others. This means that the spin current induced spin-Hall effect is the dominating effect in the present device. By analyzing the system's symmetry, the relations among these conductance matrix elements are found. The results indicated that eight matrix elements $\left(G^{x x / x y / y x / y y}\right.$ and $\left.G^{z z / z c / c z / c c}\right)$ have conserved quantities as the usual Hall effect. However, the other eight matrix elements correspond to the current simultaneously flowing out of or into two transverse terminals (as shown in the left inset of Fig. 1), which is different from the usual Hall effect. When only the Rashba SOI or the Dresselhaus SOI is present in the device, half of the matrix elements are found to be zero.

\section{ACKNOWLEDGMENTS}

This work was supported by the Knowledge Innovation Project of the Chinese Academy of Sciences and NSF-China under Grants No. 90303016, No. 10474125, and No. 10525418. J. W. is supported by RGC grant (HKU 7044/ 05P) from the government SAR of Hong Kong. 
*Electronic address: sunqf@aphy.iphy.ac.cn

${ }^{1}$ J. E. Hirsch, Phys. Rev. Lett. 83, 1834 (1999); M. I. Dyakonov and V. I. Perel, JETP Lett. 13, 467 (1971); M. I. Dyakonov and V. I. Perel, Phys. Lett. 35A, 459 (1971).

${ }^{2}$ S. Murakami, N. Nagaosa, and S.-C. Zhang, Science 301, 1348 (2003); S. Murakami, N. Nagaosa, and S.-C. Zhang, Phys. Rev. B 69, 235206 (2004).

${ }^{3}$ J. Sinova, D. Culcer, Q. Niu, N. A. Sinitsyn, T. Jungwirth, and A. H. MacDonald, Phys. Rev. Lett. 92, 126603 (2004).

${ }^{4}$ Y. K. Kato, R. C. Myers, A. C. Gossard, and D. D. Awschalom, Science 306, 1910 (2004); V. Sih, R. C. Myers, Y. K. Kato, W. H. Lau, A. C. Gossard, and D. D. Awschalom, Nat. Phys. 1, 31 (2005); V. Sih, W. H. Lau, R. C. Myers, V. R. Horowitz, A. C. Gossard, and D. D. Awschalom, Phys. Rev. Lett. 97, 096605 (2006).

${ }^{5}$ J. Wunderlich, B. Kaestner, J. Sinova, and T. Jungwirth, Phys. Rev. Lett. 94, 047204 (2005).

${ }^{6}$ S. O. Valenzuela and M. Tinkham, Nature (London) 442, 176 (2006).

${ }^{7}$ J. Shi, P. Zhang, D. Xiao, and Q. Niu, Phys. Rev. Lett. 96, 076604 (2006)

${ }^{8}$ E. M. Hankiewicz, J. Li, T. Jungwirth, Q. Niu, S.-Q. Shen, and J. Sinova, Phys. Rev. B 72, 155305 (2005).

${ }^{9}$ B. K. Nikolić, S. Souma, L. P. Zârbo, and J. Sinova, Phys. Rev. Lett. 95, 046601 (2005).

${ }^{10}$ B. K. Nikolić, L. P. Zârbo, and S. Souma, Phys. Rev. B 72, 075361 (2005).

${ }^{11}$ J. Li, X. Dai, S.-Q. Shen, and F.-C. Zhang, Appl. Phys. Lett. 88, 162105 (2006).

${ }^{12}$ In our model, a pure spin bias $V_{s}$ (with zero charge bias $V_{c}$ ) is added at the longitudinal terminals. Suppose the longitudinal terminals 1 and 3 are replaced by the ferromagnetic leads and we apply both the spin bias and the charge bias. In this case, the spin and charge currents in the transverse terminals 2 and 4 can easily be obtained from $J_{\mu}=G^{\mu \nu} V_{\nu}$ with the aid of $G^{\mu \nu}$, but now the spin current induced spin-Hall effect, the spin-Hall effect, the reciprocal spin-Hall effect and the Hall effect, will mix together.

${ }^{13}$ Q.-F. Sun, H. Guo, and J. Wang, Phys. Rev. Lett. 90, 258301 (2003); W. Long, Q.-F. Sun, H. Guo, and J. Wang, Appl. Phys.
Lett. 83, 1397 (2003); B. Wang, J. Wang, J. Wang, and D.-Y. Xing, Phys. Rev. B 69, 174403 (2004); D.-K. Wang, Q.-F. Sun, and H. Guo, ibid. 69, 205312 (2004).

${ }^{14}$ S. K. Watson, R. M. Potok, C. M. Marcus, and V. Umansky, Phys. Rev. Lett. 91, 258301 (2003).

${ }^{15}$ Under the charge bias $V_{c}$, the chemical potentials $\mu_{1 / 3}$ are spin indenpendent. So the results (e.g., the charge or spin current) are completely the same, regardless of the spin index $s$ used in $\mu_{1, s \uparrow}=\mu_{1, s \downarrow}=-\mu_{3, s \uparrow}=-\mu_{3, s \downarrow}=e V / 2$.

${ }^{16}$ Here, $G_{p}^{\mu \nu}=J_{p, \mu} / V_{\nu}$ represents $J_{p, \mu}$ divided by the bias value $V$.

${ }^{17}$ L. Sheng, D. N. Sheng, C. S. Ting, and F. D. M. Haldane, Phys. Rev. Lett. 95, 136602 (2005); J. Li, L. Hu, and S.-Q. Shen, Phys. Rev. B 71, 241305(R) (2005).

${ }^{18}$ C. P. Moca and D. C. Marinescu, Phys. Rev. B 72, 165335 (2005).

${ }^{19}$ Here, the four leads are also described by the discrete tightbinding Hamiltonian, which is the same as that in Ref. 17.

${ }^{20}$ Electronic Transport in Mesoscopic Systems, edited by S. Datta (Cambridge University Press, England, 1995), Chaps. 2 and 3; T. P. Pareek, Phys. Rev. Lett. 92, 076601 (2004).

${ }^{21}$ F. Mireles and G. Kirczenow, Phys. Rev. B 64, 024426 (2001); Q.-F. Sun, J. Wang, and H. Guo, ibid. 71, 165310 (2005).

${ }^{22}$ In Ref. 10, the matrix element $G^{x c}$ is very small, but it is not zero. This is different from the our present results $\left[G^{x c}=0\right.$ exactly, see Table I(a)], although the system is completely the same. They may have neglected a factor of $G_{22}^{\text {out }}-G_{22}^{\text {in }}$ in their Eq. (8).

${ }^{23}$ Notice that $G_{2(4)}^{z c}=G_{2(4)}^{c z}$ is valid only for the square scattering center region. For the rectangular scattering center region, $G_{2(4)}^{z c}$ is in general not equal to $G_{2(4)}^{c z}$.

${ }^{24}$ Y.-S. Gui, C. R. Becker, N. Dai, J. Liu, Z.-J. Qiu, E. G. Novik, M. Schäfer, X.-Z. Shu, J.-H. Chu, H. Buhmann, and L. W. Molenkamp, Phys. Rev. B 70, 115328 (2004).

${ }^{25}$ Here, the parameters of the material " $n$-type $\mathrm{HgTe}$ " are used in the numerical calculation, which is the same as that in Ref. 24. In fact, for other materials (e.g., GaAs with $m^{*}=0.036 m_{e}$ ), the numerical results are similar.

${ }^{26}$ Y. Xing, Q.-F Sun, and J. Wang, Phys. Rev. B 73, 205339 (2006).

${ }^{27}$ L. Sheng, D. N. Sheng, and C. S. Ting, Phys. Rev. Lett. 94, 016602 (2005). 